\title{
Ein Konzept für den energieeffizienten Betrieb von Mobilfunknetzen
}

\author{
N. Bayer and D. von Hugo \\ Deutsche Telekom AG Innovation Laboratories, Darmstadt, Deutschland \\ Correspondence to: D. von Hugo (dirk.von-hugo@telekom.de)
}

Received: 7 November 2014 - Revised: 11 February 2015 - Accepted: 26 February 2015 - Published: 3 November 2015

\begin{abstract}
Kurzfassung. Der flächendeckende Betrieb mehrerer Mobilfunknetze unterschiedlicher Technologie in einem Land sorgt aufgrund der ständigen Bereithaltung von Übertragungskapazität für Dienste mit zunehmend höherem Datenvolumenbedarf für einen erheblichen Energieverbrauch. Das Forschungsförderungsprojekt ComGreen hat sich zur Aufgabe gesetzt, durch lastadaptiven Betrieb und intelligente dynamische Rekonfiguration des Funkzugangsnetzes zur Energieeinsparung beizutragen. Konzept, Herausforderungen, ausgewählte Ergebnisse von Simulationen und prototypischem Betrieb werden ebenso wie typische Erwartungswerte des künftigen Energieverbrauchs im Mobilfunkbereich vorgestellt. Sowohl Berechnungen als auch Messungen zeigen, dass durch kontext-basierte dynamische Rekonfiguration von zellularen Funknetzen Energieeinsparungen im Bereich von 25-40\% ermöglicht werden.
\end{abstract}

\section{Einleitung}

Angesichts des erwarteten stetig steigenden Bedarfs nach breitbandiger mobiler Datenübertragung (Cisco, 2014) wird auch ein stark zunehmender Energieverbrauch im ICTBereich prognostiziert, was der versprochenen Energieeinsparung durch Green IT (Grüne Informationstechnologie, d. h. Klimaschutz durch Vermeidung von Reisen und Materialtransport, effizientere Steuerung von Stromverbrauchern usw.) zuwiderlaufen würde. Ein Großteil der elektrischen Energie bei Anbietern von Mobilfunkdiensten wird in den Funknetzen verbraucht, die heutzutage in der Regel ständig überall maximale Übertragungskapazitäten bereitstellen, obwohl die tatsächliche Nachfrage zeitlich und örtlich stark schwankt. Das vom Bundesministerium für Wirtschaft und Energie (BMWi) im Rahmen des Programmes IT2Green (BMWi, 2014) geförderte Forschungsprojekt
ComGreen (Communicate Green; ComGreen, 2014) konnte zeigen, wie durch lastadaptiven Betrieb (Lange et al., 2014) und Einsatz von verbesserter intelligenter Hard- und Software im Mobilfunkzugang mindestens $30 \%$ des heutigen Energieverbrauchs eingespart werden kann. Dabei wurden nicht nur Algorithmen entwickelt, um Basisstationen der neuesten Funktechnologien bedarfsabhängig ganz oder teilweise abschalten zu können, sondern auch Mechanismen untersucht, wie das Transportnetz und die Gesamtnetzsteuerung effizienter betrieben werden können. Wie das ohne Beeinträchtigung der Kundenzufriedenheit geschehen kann, wurde im Projekt ebenfalls teilweise untersucht.

Ansatzpunkt ist die selbstorganisierte, kontextbasierte Anpassung der Betriebsparameter eines heterogenen Funknetzes, so dass bestmöglich zwischen Energieaufwand und erzielter Dienstgüte abgewogen werden kann. Durch Systemsimulationen und die Einrichtung und den experimentellen Betrieb von entsprechenden Versuchsaufbauten (sog. Demonstratoren) konnte die Wirksamkeit der vorgeschlagenen Konzepte und Algorithmen zur Steuerung der Netzkonfiguration nachdrücklich bestätigt werden (Gomez et al., 2013). Die zu erwartenden Auswirkungen eines solchen energieeffizienten Netzes auf den Energieverbrauch im Endgerät des Kunden wurden unter Berücksichtigung verschiedener Dienstnutzungen und Nutzerprofile für typische Energieverbrauchsmodelle von Mobiltelefonen ebenfalls untersucht. Auf Grundlage von Modellen zur Bestimmung der vom Nutzer empfundenen Dienstqualität als Funktion der Netzqualität (d.h. im Wesentlichen der Paketverlustrate und Übertragungsverzögerung) wurde ermittelt, dass nur eine unmerkliche temporäre Verschlechterung der Dienstqualität bei erheblichen Energieeinsparungen zu erwarten ist.

Abschätzungen des künftig zu erwartenden Energieverbrauchs im Mobilfunksektor unter der Annahme eines wei- 
ter steigenden Bedarfs nach mobiler Übertragungskapazität schwanken in Abhängigkeit von den unterstellten Szenarien um bis zu $50 \%$. Durch den Einsatz neuer Mechanismen zur Verbesserung der Energieeffizienz können aber in jedem Fall deutliche Einsparungen beim Energieverbrauch erzielt werden. Die entwickelten Verfahren tragen damit zur Senkung der Betriebskosten bei und helfen, den Ausstoß der sog. Treibhausgase $\mathrm{zu}$ verringern.

Der Beitrag gliedert sich im Weiteren wie folgt: Nach einer Motivation für die gestellte Aufgabe in Kapitel 2 und einem Überblick zum Stand der Technik werden in Kapitel 3 die erzielten Ergebnisse in ausgewählten Arbeitspaketen dargestellt. In Kapitel 4 werden die untersuchten Verfahren auf den zukünftig zu erwartenden Mobilfunkverkehr angewendet, um die Auswirkungen für unterschiedliche Szenarien zu quantifizieren. Eine Zusammenfassung und Bewertung der erzielten Ergebnisse wird in Kapitel 5 gegeben.

\section{Motivation}

Da mit dem eingangs genannten steigenden Volumen an über Mobilfunk übertragenen Daten keine adäquate Steigerung des damit erzielten Umsatzes einhergeht, wie aus veröffentlichten Zahlen der Bundesnetzagentur (BNetzA, 2014) ersichtlich wird, im Gegensatz dazu aber eine Steigerung der Energiekosten (wie auch der Folgekosten eines übermäßigen Energieverbrauchs) zu erwarten ist, ist die Verbesserung der Energieeffizienz - wie in fast allen Lebensbereichen auch - unabdingbar notwendig. Abbildung 1 zeigt auf der linken Seite eine Gegenüberstellung des nach Prognosen von Cisco (2014) und unter Annahme eines moderaten Umsatzwachstums fortgeführten und bis 2020 für Deutschland zu erwartenden mobilen Datenvolumens in TB und des entsprechenden Umsatzes in Mrd. €. Zusätzlich ist die Prognose des Projektes Greentouch (2013) eingefügt, die einen um mindestens das Dreifache höheren Datenbedarf voraussagt. Auf der rechten Seite der Abbildung 1 ist der für den gleichen Zeitraum erwartete Energieverbrauch im Mobilfunk aufgetragen zusammen mit den bei zwei verschiedenen Preissteigerungsraten ( 3 bzw. $5 \%$ ) anzunehmenden entsprechenden Kosten (ausgehend von 0.25 $€$ pro kWh in 2008). Dabei berücksichtigt die jährliche Steigerung im Energieverbrauch von ca. $5 \%$ bereits technische Verbesserungen der Funksystemkomponenten und eine höhere Effizienz in pro übertragenem Byte benötigter Energie. Trotzdem ist mit deutlich gesteigerten Energiekosten innerhalb von 12 Jahren zu rechnen.

Wie in Abbildung 2 anhand der Größenordnung der Anzahl der jeweils beteiligten Komponenten in den einzelnen Netzbereichen und der Größenverhältnisse des jeweiligen Energieverbrauchs zu erkennen, wird der überwiegende Anteil der Energie im Funkzugangsnetz verbraucht. Die dargestellten relativen Werte sind vergleichbar, unabhängig ob man die von NSN weltweit geschätzten Zahlen (Scheck, 2013) unterstellt oder die vom Projekt ComGreen unter An- nahme eines konvergenten Netzbetriebes von Festnetz- und Mobilfunk (Fixed Mobile Convergence, FMC) ermittelten Verhältnisse. Dabei ist zu berücksichtigen, dass Komponenten im Funkzugang (RAN, Radio Access Network) und Kernnetz (CN, Core Net) in der Regel ausschließlich für Mobilfunk genutzt werden, während das Aggregationsnetz (Agg) - besonders im Blick auf FMC - sowohl Mobilfunkals auch Festnetzverkehr transportiert. Letzteres trifft zunehmend auch für den Backbone, also das Transportnetz zwischen den CN-Komponenten, zu. Da mögliche Einsparungen im RAN sich absolut am stärksten auswirken, liegt der Forschungsschwerpunkt hier auf dem energieeffizienten Management komplexer heterogener Zugangsnetze für den Mobilfunk. Allerdings werden auch Optimierungen im Kernnetz untersucht, das eine Steuerung verschiedener Zugangsnetze und Verwaltungsfunktionen wahrnimmt sowie den Übergang in andere (Fest- und Mobilfunk-) Netze und die Zusammenschaltung mit weltweiten Netzknoten realisiert. Der Lösungsansatz von ComGreen liegt dabei auf einer intelligenten Steuerung der beiden Netzbereiche eines Betreibers, wobei die Kontextinformation (z.B. die aktuelle Anzahl und Verteilung der Nutzer in Netzen, die Art von Diensten, die momentan von Kunden genutzt werden, Vorhersage zur Intensität der Netzwerkauslastung usw.) Grundlage der Entscheidungen ist. Basierend auf vorhandener und systematisch aufbereiteter Kontextinformation können bestimmte Operationen angestoßen werden, die zur Reduzierung des Energieverbrauchs führen werden.

\subsection{Prinzip des lastadaptiven Netzbetriebes}

Das Prinzip eines lastadaptiv betriebenen Mobilfunknetzes ist die möglichst exakte dynamische Anpassung der angebotenen Funknetzkapazität an den aktuellen Bedarf, also die tatsächlich nachgefragte Übertragungsrate. Die Wirkungsweise einer solchen Anpassung ist in Abbildung 3 dargestellt, wo der Tagesverlauf von Übertragungskapazität, Leistungsbedarf und Energieverbrauch schematisch (in relativen Einheiten) dargestellt ist. Links (a) wird der variable nachgefragte Datenbedarf der herkömmlich üblicherweise (annähernd) konstant angebotenen Kapazität gegenübergestellt sowie der, die sich für ideale und realistische (granulare) lastadaptive Schaltung (wie von ComGreen angestrebt) der jeweils benötigten Netzressourcen (Bandbreite, Sendeleistung) ergibt. In der mittleren Grafik (b) ist die entsprechend aufgenommene (hier: direkt proportionale) Leistung für den herkömmlichen und gewählten realistischen Ansatz sowie eine für die Rekonfigurationen benötigte zusätzliche Schaltleistung über $24 \mathrm{~h}$ aufgetragen, während rechts (c) der aufsummierte Energieverbrauch über der Zeit für die vier Ansätze dargestellt wird. Die Rekonfigurationen sind hier jeweils durch (grüne) Dreiecke symbolisiert. Dabei spielt neben der Granularität der Kapazitätszuschaltung (hier: in zehn gleichmäßigen Schritten), dem für eine Konfiguration benötigten Zeitaufwand $\tau$ (hier: $1 \mathrm{~h}$ ) und dem Abstand zwi- 

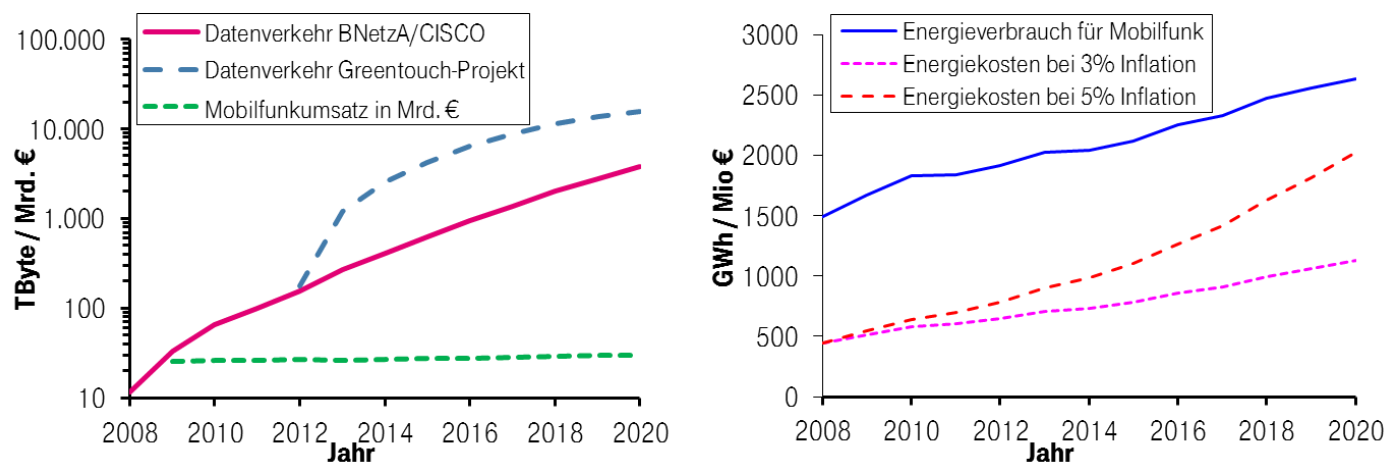

Abb. 1. Entwicklung von mobilem Datenverkehr (wie von Cisco bzw. dem Projekt Greentouch erwartet) und entsprechendem Umsatz in Deutschland (links) sowie der zugehörige Energieverbrauch und dessen Kosten bei Annahme von zwei Preissteigerungsraten (rechts).
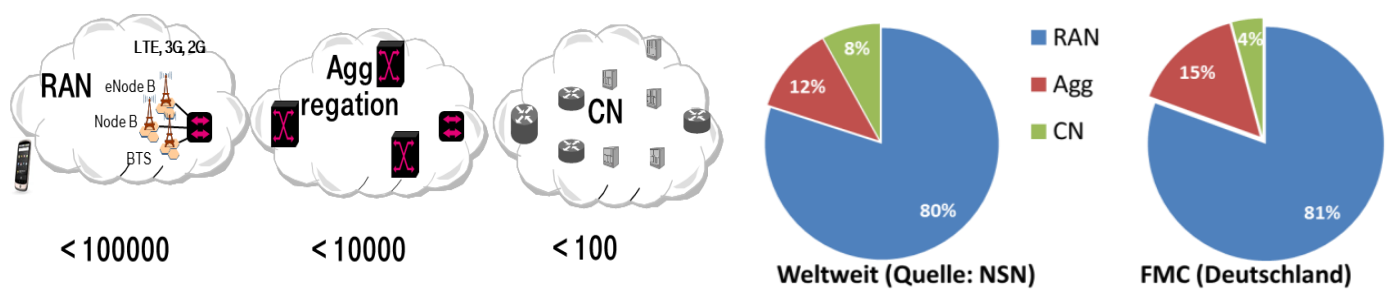

Abb. 2. Prinzipieller Aufbau des Mobilfunknetzes (links) mit Funkzugang (RAN), Aggregation (Agg) und Kernnetz (CN) mit der zahlenmäßigen Größenordnung der beteiligten Netzknoten sowie dem relativen Anteil des Energieverbrauchs (rechts) unter zwei Annahmen.

schen zwei Konfigurationen $\delta$ (z.B. 4 h) auch die Verzögerung für die Erstellung der Verkehrsprognose aus Kontextdaten $\varepsilon$ (in Abbildung 3 nicht dargestellt) eine wesentliche Rolle bei der erzielbaren Energieeinsparung. Durch Messungen im Multi-RAT-Testbed wurden z.B. für ein WLAN IndoorNetz folgende Werte ermittelt: $\tau=61 \mathrm{~s}, \varepsilon=35 \mathrm{~s}$ bei einer Schaltleistung von 37 pro $620 \mathrm{~mW}$ Nutzleistung (pro IndoorFunkschnittstelle), also $6.0 \%$. Im Outdoor-Fall war der relative Leistungsbedarf für Rekonfigurationen mit $11.6 \%$ ebenso wie der Zeitaufwand $\tau$ mit $120 \mathrm{~ms}$ fast doppelt so groß (Bayer et al., 2014), was z.T. auch an der höheren Komplexität der Geräte liegt, die im Außenbereich zum Einsatz kommen.

Im in Abbildung 3 gezeigten Beispiel würde bei idealer Adaption nur die halbe Energie verbraucht, bei verlustfreier Umschaltung durch ComGreen-Prozeduren eine Energieeinsparung von $31.3 \%$ bzw. unter Berücksichtigung der angenommenen Rekonfigurationsleistung von $25.4 \%$ erzielt. Eine detaillierte Untersuchung der Abhängigkeiten zwischen erzielbarer Energieeffizienz und den verschiedenen Parametern, die den Konfigurationsprozess charakterisieren, findet sich in (Kaus, 2012) und (ComGreen, 2013). Dabei wurden im Simulator die Schwellwerte für das Zuschalten zusätzlicher und das Abschalten nicht mehr benötigter Ressourcen (z.B. zwischen 40-90\% der verfügbaren und $10-50 \%$ der abzuschaltenden Kapazität) variiert sowie zentrale und dezentrale Entscheidungsalgorithmen simuliert, und die Energieeinsparung sowie Degradation in Form fehlender Band- breite ermittelt. Weiterhin wurde die optimal erreichbare Effizienzsteigerung als Funktion des Schaltaufwandes (benötigte Energie pro geschalteter Kapazitätseinheit), der Granularität der Schaltstufen und des zeitlichen Abstandes zwischen aufeinanderfolgenden Rekonfigurationen berechnet.

\subsection{Stand der Technik}

Das Thema Energieeffizienz in Telekommunikationsnetzen hat in den letzten Jahren zunehmend an Bedeutung gewonnen - sowohl in der öffentlichen Wahrnehmung als auch im akademischen und industriellen Forschungs- und Entwicklungsumfeld. Optimierung von Funknetzen dagegen ist seit Beginn der kommerziellen Nutzung zur effizienten Planung und Betrieb unerlässlich gewesen, wobei der Hauptaugenmerk auf der Netzqualität liegt, die sich in Form von flächendeckender Versorgung und Kapazität, aber auch von Verfügbarkeit und Verlässlichkeit, Fairness bei Ressourcenzuweisung usw. beschreiben lässt.

Eine umfassende Studie zu Zusammenhang und Entwicklung von Umweltauswirkungen durch IKT (Informationsund Kommunikationstechnik) wurde im Jahr 2008 veröffentlicht (The Climate Group/GeSI, 2008). Hier wurde das größte Potential für einen positive Einfluss bis 2020 darin gesehen, durch IKT die Energieeffizienz bei denjenigen industriellen Prozessen zu erhöhen, die zu den Quellen für starke Treibhausgasemissionen zählen, wie Energieerzeugung und -verteilung, Gebäudetechnik, produzierende Industrie 


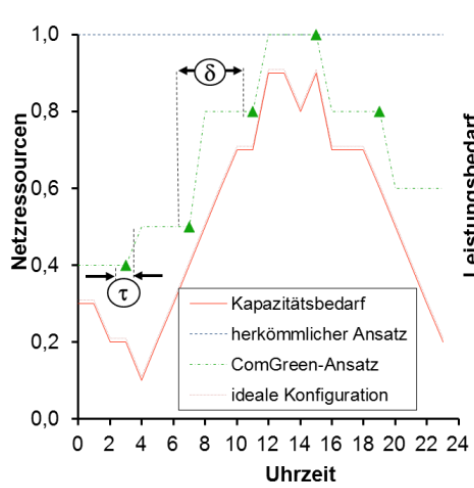

a) Kapazitätsbedarfund Angebot

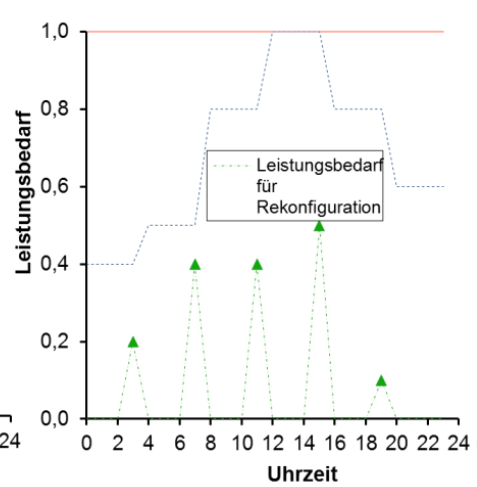

b) Leistungsaufnahme

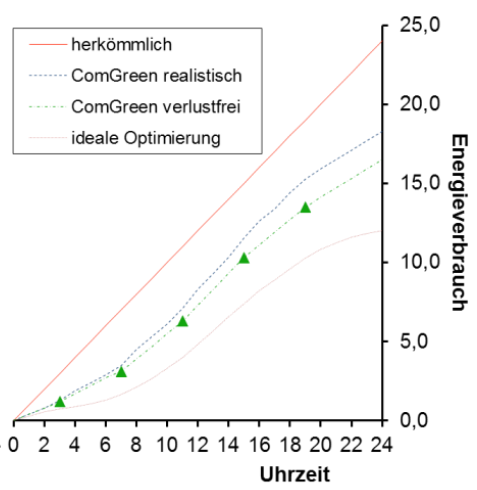

c) Kumulierter Energieverbrauch

Abb. 3. Bereitgestellte Netzkapazität, erforderliche Leistung und der sich ergebende entsprechende Energieverbrauch im exemplarischen Tagesverlauf.

und Transport. Weiterhin wird betont, dass die Fortsetzung der derzeit beobachteten stetigen Zunahme des mobil genutzten Datenvolumens sehr bald dazu führen würde, dass mobile und drahtlose Übertragungssysteme den überwiegenden Teil des IKT Energieverbrauchs ausmachen. Der Bericht der OECD (2009) sieht den Beitrag von IKT neben dem (hauptsächlich elektrischen) Energieverbrauch auch in umweltschädlichen Emissionen bei Herstellung, Transport und Entsorgung von IKT-Geräten (Netzkomponenten wie Endgeräten). Statt des Ansatzes, den gesamten Lebenszyklus zu betrachten (Total Life Cycle Analysis) wird im Folgenden nur die in der Nutzungsphase (Betrieb) benötigte Energie betrachtet.

Unter Berücksichtigung auch des Kostendrucks auf die Mobilfunkbetreiber und der Verantwortung gegenüber der Gesellschaft im Hinblick auf die ökologischen und klimatischen Auswirkungen wurden verschiedene Aktivitäten zur Energieeinsparung bei zellulären und lokalen Funknetzen gestartet. 3GPP (3rd Generation Partnership Project) hat im Rahmen der Initiative SON (Selbst-Organisierende Netze) den Anwendungsfall Energy Saving (ES) definiert und begonnen, die Standardisierung der nächsten Mobilfunktechnik E-UTRAN (Enhanced UMTS Radio Access Network) mit der Luftschnittstelle LTE (Long Term Evolution) mit Blick auf Energieeffizienz zu erweitern (3GPP, 2011). Mehrere EU-Projekte haben Aspekte der Energieeffizienz im Zusammenhang mit SON-Verfahren für $2 \mathrm{G}, 3 \mathrm{G}$ und LTE (sowie LTE-Advanced), aber auch für WLAN und andere Kurzstrecken-Funktechnik, untersucht.

Im Projekt OPERA-Net (Optimising Power Efficiency in mobile RAdio Networks, 2014) wurde festgestellt, dass Energieeinsparungen von $33 \%$ erreichbar sind, wenn einzelne Sektorantennen einer Basisstation in einen Schlafmodus geschaltet werden können. Das EU-geförderte Integrierte Projekt E3 (End-to-End-Efficiency, 2009) hat quantifiziert, dass durch die SON-Anwendung ES Betriebskosten um 20-40\% reduziert werden können, ohne die Dienstgü- te einzuschränken (wobei entweder die Sendeleistung reduziert oder eine komplette Basisstation ausgeschaltet wird). Projekt EARTH (Energy-Aware Radio and neTwork tecHnologies) wiederum hat sich ausführlich mit Modellen und Algorithmen befasst, die den Energieverbrauch von Funknetzen bei geringer Last erheblich zu reduzieren vermögen und neue Netzmanagement-Konzepte für dynamische Re-Konfiguration von Mobilfunknetzen (z. B. Selbstoptimierung, automatische Konfiguration und Standby-Betrieb) entworfen. Es wird erwartet, dass dadurch eine durchschnittliche Verbesserung der Energieeffizienz von $40 \%$ erzielt werden kann (Correia et al., 2010). NoE (Network of Excellence) TREND (Towards Real Energy-efficient Network Design) hat sich eine Quantifizierung des Energiebedarfs gegenwärtiger und künftiger Telekom-Infrastrukturen zur Aufgabe gemacht. Das Projekt beschäftigt sich mit energieeffizienten, skalierbaren und nachhaltigen zukünftigen Netzen und hat in diesem Rahmen Maßnahmen wie z. B. Strategien zum Ein- und Ausschalten von Netzkomponenten, energieeffiziente Planung und Steuerung von Netzen usw. untersucht (Le Rouzic et al., 2013).

Im Rahmen der weltweiten Standardisierungstätigkeit von 3GPP wurden verschiedene Szenarien und Simulationen der Energieeinsparung erstellt, deren Ergebnisse zwischen 18 und $38 \%$ (3GPP, 2010) variieren. Innerhalb eines Dokuments zu ,grünen Aktivitäten“ bei den aktuellen Releases von 3GPP (ETSI MCC, 2012) wird die Bedeutung der Energieeffizienz sowohl in Bezug auf Kosten als auch die Umwelt betont. Aufgrund der hohen Anforderungen von Betreibern an die Steuerung und Überwachung von Energiesparfunktionen und die Bewertung ihrer Auswirkungen auf die Qualität der Netze und Dienste wird ein entsprechendes effizientes und standardisiertes Netzmanagement benötigt.

Einen umfassenden Überblick über aktuelle Themen und Herausforderungen in der Forschung zur Energieeffizienz in Mobilfunknetzen gibt (De Domenico et al., 2014). 


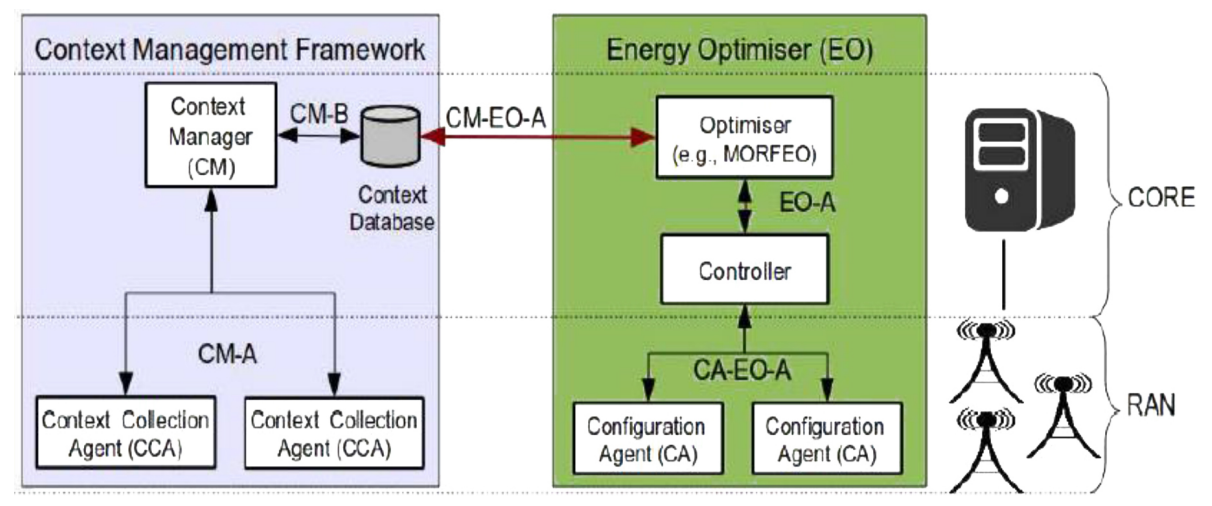

Abb. 4. Funktionale Architektur des kontext-basierten ComGreen-Konzeptes.

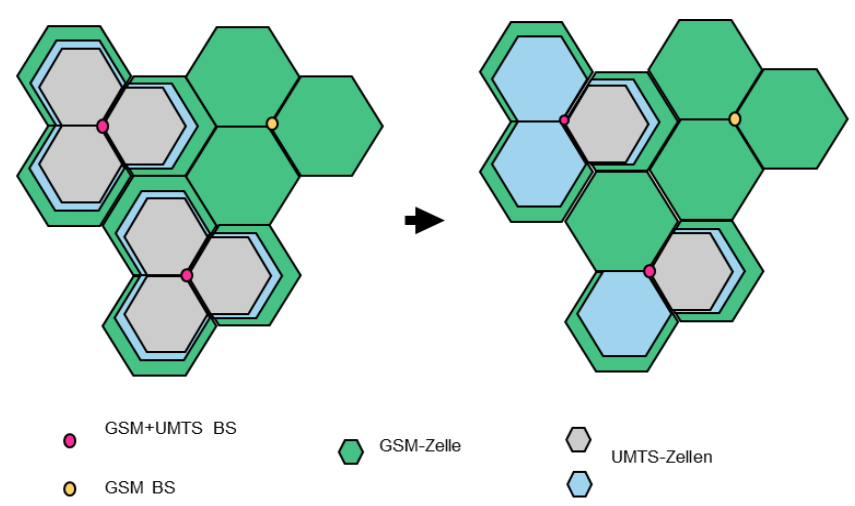

Abb. 5. Prinzipielle Darstellung von „Multi-RAT macro cell sleep mode" (Schlafmodus für Makrozellen im Fall mehrerer Funktechniken).

\section{Ergebnisse}

\subsection{Architektur}

Die adaptive, kontextabhängige und Technologieübergreifende Systemarchitektur für eine effiziente Steuerung des Energieverbrauchs in heterogenen Funknetzen wird in (Bayer et al., 2011) beschrieben. Die Entwicklung einer Kontext-Einheit, die Informationen aus verschiedenen Quellen, die im Kontext zur Nutzung des Mobilfunkdienstes stehen, sammelt und verarbeitet, um kontextbezogen aus der Datenbasis Entscheidungen für die aktuelle Netzkonfiguration abzuleiten, wird in (Uzun et al., 2011, 2014) detailliert. Einen Überblick über die neuartige Systemarchitektur zur Nutzung kontextbezogener Information liefert Abbildung 4. Die logische Architektur besteht im Wesentlichen aus zwei Bausteinen: dem Kontext-Management-Framework und dem Energie-Optimierer (EO). Ersterer umfasst mehrere Funktionselemente und dient zur zentralen Sammlung (CCA, Context Collection Agent), Verarbeitung und Verwaltung (CM, Context Manager) der Informationen. Diese kontextbezogenen Daten werden in einer Datenbank (Context
Database) gespeichert und genutzt, um mit Hilfe des Optimierungsmodules den aktuellen und künftigen (optimierten) Zustand des Netzes und seiner Elemente zu berechnen. Die Optimierung erfolgt so, dass der Kapazitätsbedarf der Kunden mit dem jeweils geringstmöglichen Energieverbrauch erfüllt werden kann. Mit Hilfe der errechneten Parameter werden über den Controller die Netzbereiche oder ggf. sogar jede einzelne Basisstation über die lokal etablierte Funktion eines Configuration Agent (CA) zur Umsetzung der entsprechenden Rekonfigurierung angesteuert. Durch Vergleich der wiederum vom CCA gemessenen Netzperformanz mit den Zielwerten wird der Regelkreis zur Überwachung des „ordnungsgemäßen“ Netzbetriebes geschlossen.

\subsection{Verfahren}

Maßnahmen, die zu einer höheren Energieeffizienz beitragen können und im Rahmen des Projektes ComGreen untersucht wurden, folgen im Wesentlichen einem der nachfolgend genannten Prinzipien:

Bei Einbuchung eines Endgerätes in das Netz oder einem anstehenden Zellwechsel wird die Entscheidung zugunsten einer Verbindung zur energetisch günstigsten Zelle/Basisstation getroffen. Diese Strategie erfordert eine entsprechende ,policy“ und kann auf alle nicht homogenen Netzstrukturen angewendet werden, auch wenn z.B. nur eine einzige Funktechnik wie GSM genutzt wird. Durch unterschiedliche Alterung der Hardware, aber auch durch Unterschiede in der aktuellen Auslastung einer Station oder auch der räumlichen Verteilung der von ihr versorgten Kunden weicht der spezifische Energieverbrauch der einzelnen Verbindungen voneinander $\mathrm{ab}$. Da oft mehrere Zellen als Kandidaten bereitstehen, die alle den Anforderungen an ausreichende Übertragungsqualität genügen, ist eine solche Vorgehensweise eines ,energy efficient connectivity management“" mit relativ wenig Aufwand realisierbar.

Eine Anpassung des Übertragungsmodus an den Bedarf ist theoretisch unter Ausnutzung des Shannon-Theorems möglich, wonach die Kanalkapazität linear zur Bandbreite ist, 
aber logarithmisch von der Sendeleistung abhängt: Wird bei einer nicht voll ausgelasteten LTE-Zelle (nicht alle Resource Blocks werden genutzt) die Bandbreite erhöht und dafür die Leistung reduziert, so lässt sich mit Hilfe dieses „Bandwidth Expansion Mode“"(BEM) Energie einsparen.

Schließlich besteht die klassische Methode darin, nicht benötigte Hardware ,einfach“ abzuschalten (d.h. kontrolliert in einen wenig bis keine Energie verbrauchenden Schlafmodus zu versetzen): dies betrifft einzelne Komponenten einer Station wie Leistungsverstärker, Träger, Sektoren, usw. bis hin zu einem kompletten Standort. Das Verfahren kann für alle Techniken angewendet werden, sofern die Aus- und Einschaltung ohne Probleme (Reduzierte Lebensdauer, Risiko eines Defektes o.ä.) möglich ist. Hier wird GSM ausdrücklich ausgenommen, um eine Grundversorgung kontinuierlich bereitzustellen und da eine entsprechende Erweiterung der Schaltmöglichkeit bei der weitgehend ausgereiften Technik nicht zu erwarten ist.

Außer Betracht bleibt hier die Untersuchung eines veränderten Kunden- oder Nutzungsverhaltens - etwa mit dem Ziel, die Lastadaption in Form einer gleichmäßigeren Auslastung der Zellen durch Gleichverteilung des Datenbedarfs zu erreichen. Denkbar wären Anreize, um in den Verkehrsspitzen (oder aber auch in der Nacht) auf eine bandbreiteintensive Nutzung zu verzichten. Tatsächlich können solche Maßnahmen die Energieeffizienz steigern und sind Gegenstand anderer Untersuchungen (Hsieh et al., 2014), hier sollen aber nur netzbezogene Verfahren betrachtet werden. Eine an anderer Stelle durchgeführte Kundenbefragung bestärkte zudem die Vermutung, dass Kunden selbst bei ausgeprägtem Umweltbewusstsein mehrheitlich nicht gewillt sind, im Bereich Mobilkommunikation Einschränkungen hinzunehmen oder Mehrkosten aufzuwenden (Deutsche Telekom AG, 2011).

Die durch Entwicklung entsprechender Strategien und Algorithmen nachgewiesene Möglichkeit eines energieeffizienteren Mobilfunknetzbetriebes kann einen Netzbetreiber darin bestärken, solche Verfahren im Wirkbetrieb einzuführen. Allerdings wird schon aus den im vorangegangenen Abschnitt abgeschätzten Werten deutlich, dass bestimmte System-spezifische Parameter wie Schnelligkeit und Energiebedarf der Schaltprozesse die erzielbare Einsparung stark beeinflussen und eine entsprechende Weiterentwicklung der Funktechnik-Komponenten notwendig wird. Hier wird es Aufgabe der Regulierer und von entsprechenden Vertragsverhandlungen sein, technisch mögliche Charakteristika auch in spezifizierte Anforderungen zu übernehmen.

\subsection{Berechnung und Experiment}

Im Folgenden werden anhand ausgewählter Verfahren quantitative Resultate aus Simulationen und analytischen Berechnungen exemplarisch vorgestellt sowie die Auswertung von Messungen an experimentellen Aufbauten von ComGreen präsentiert.

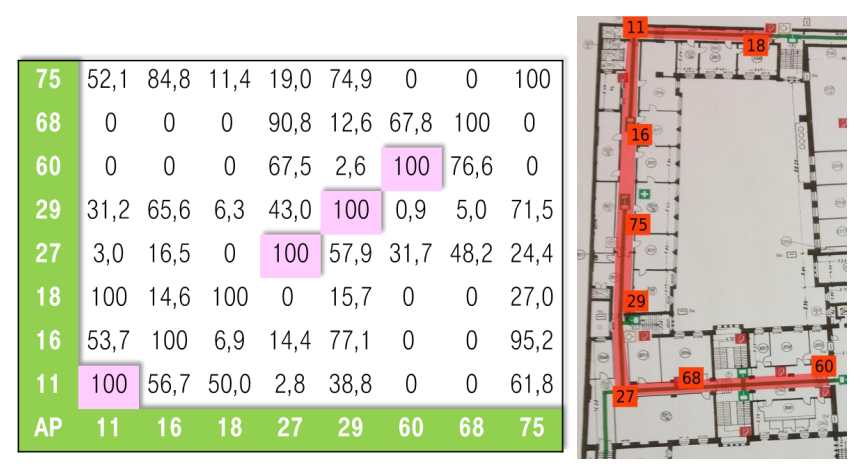

Abb. 6. Tabelle der Nachbarschaftsbeziehungen (links) mit der prozentualen Wahrscheinlichkeit einer gleichzeitigen Sichtbarkeit der APs, deren Anordnung in einem Bürogebäude rechts gezeigt wird.

Im Beispiel des Multi-RAT-Makrozellen-Schlafmodus (Multi-RAT macro cell sleep mode) besteht eine Anwendung für Kontextinformation darin, Aussagen zur Möglichkeit eines störungsfreien Abschaltens einer Makrozelle zu liefern: Wird ein aktives Funksignal abgeschaltet, das über eine Sektorantenne eine Zelle aufspannt, muss in diesem Gebiet eine Versorgung durch andere Zellen weiterbestehen, die verbliebene oder neue Kunden aufnehmen können. Dabei werden zwei Fälle unterschieden:

Im Fall ko-lokalisierter Stationen, d.h. wenn am gleichen Standort Antennen mit mehreren Funktechnologien (z.B. GSM und UMTS) bestückt sind, ist in der Regel ein unterbrechungsfreies Abschalten möglich, da sich die Versorgungsgebiete hier weitgehend decken. In Abbildung 5 ist dieses Szenario dargestellt. Auf der linken Seite ist ein Netzbereich, bestehend aus $3 \times 3 \times 3=27$ Zellen gezeigt, in dem im Normalfall (d.h. ohne Optimierung) alle drei Basisstationen, alle drei Sektoren je Station und alle drei Träger (zwei Kanäle für UMTS in blau und grau, ein GSM-Signal in grün) eingeschaltet sind (z.B. wenn in der sog. „Peak hour“" Lastspitzen auftreten). Auf der rechten Seite ist das für Zeiten schwacher Auslastung um-konfigurierte (optimierte) Netzwerk dargestellt, in dem die bereitgestellte Kapazität durch Ausschalten von Funkkomponenten reduziert wird.

Im Fall nicht ko-lokalisierter Stationen - also in Abbildung 5 (rechts) für die mittlere Zelle, in der UMTS vollständig abgeschaltet wurde und die „nördliche“ Nachbarzelle mit beiden aktiven UMTS-Trägern von einer anderen Basisstation gebildet wird - ist die Kenntnis der Nachbarschaftsbeziehungen notwendig. In diesem Beispiel handelt es sich um die Kenntnis der Versorgungswahrscheinlichkeit im Bereich der abgeschalteten UMTS-Zelle durch die benachbarten fünf Zellen, von denen drei zu anderen Standorten gehören. Ist diese Wahrscheinlichkeit, dass Endgeräte im Bereich der unteren GSM+UMTS-Basisstation auch die anderen Stationen „sehen“, ausreichend groß, kann eine Makrozelle abgeschaltet werden. Solche Nachbarschaftsbeziehungen können in Form einer „Nachbarschaftstabelle“ veran- 

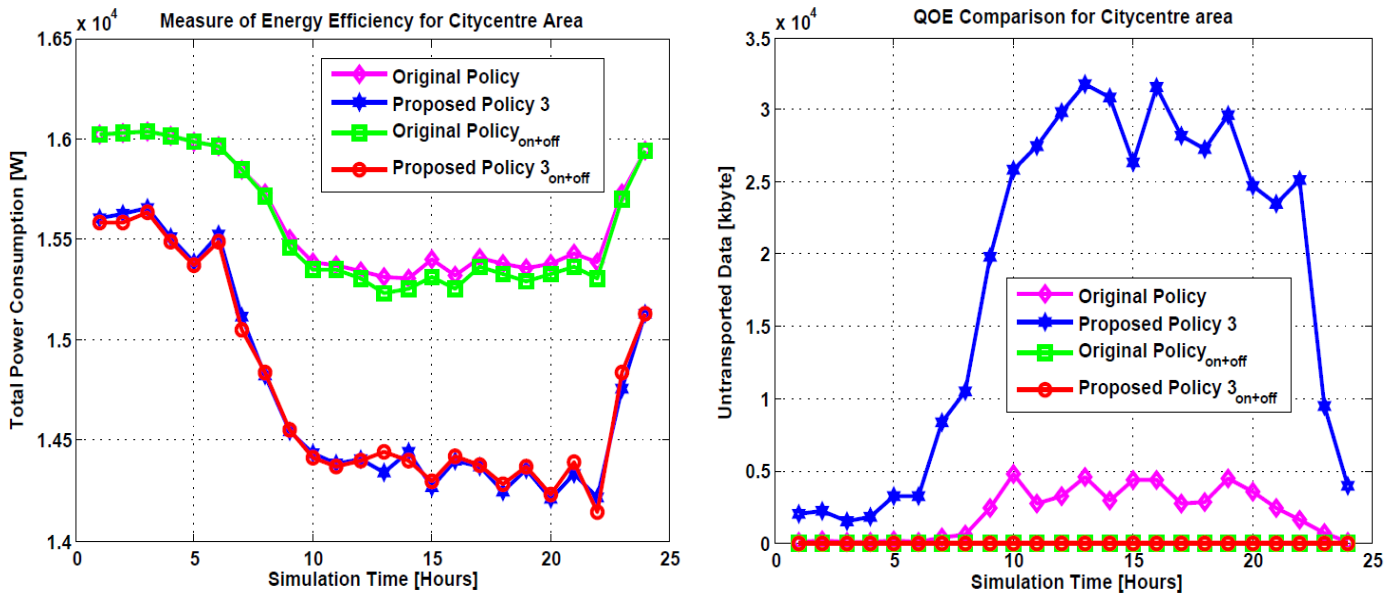

Abb. 7. Ergebnisse der Systemsimulation für Connectivity Management für verschiedene Strategien: Leistungsbedarf (links) und Qualitätsauswirkung in Form von mangels Kapazität nicht übertragenen Daten (rechts) sind im Tagesverlauf dargestellt.

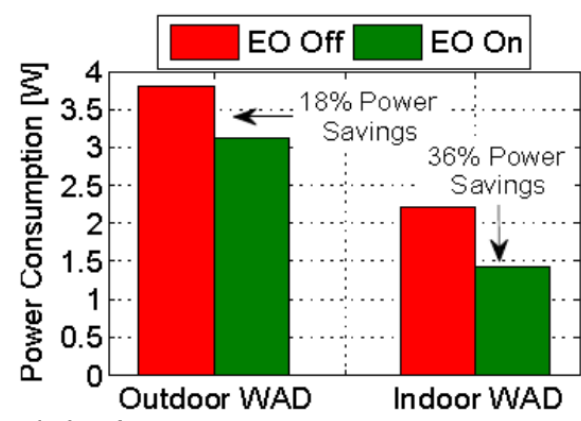

a) ohne Last

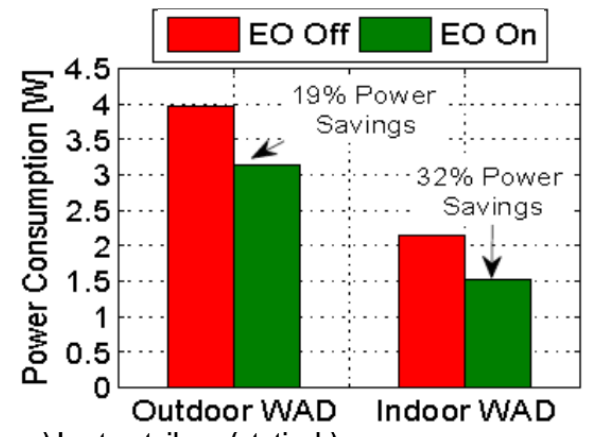

c) Lastverteilung (statisch)

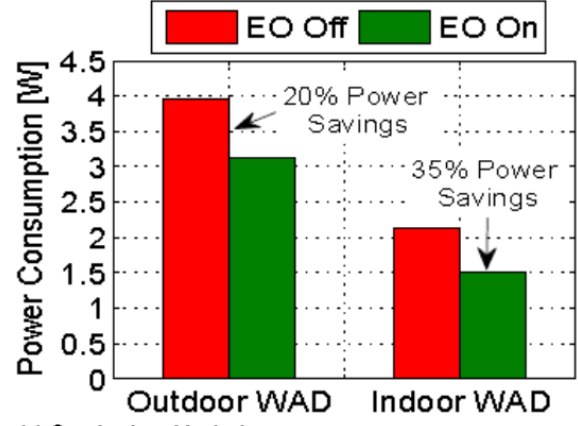

b) Statischer Verkehr

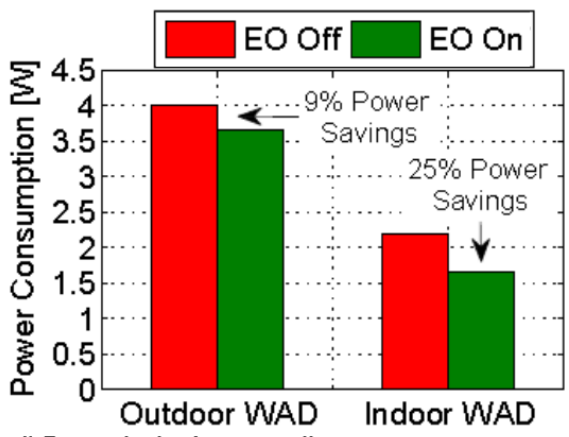

d) Dynamische Lastverteilung

Abb. 8. Messergebnisse der mittleren Leistung in W pro AP mit und ohne Energieoptimierung (EO) durch Morfeo-Algorithmus für verschiedene Szenarien und Stationen in und außer Haus.

schaulicht werden. Für eine Gruppe von acht benachbarten WLAN-Zugangsknoten (Access Points, APs) wurde eine aus der entsprechenden Messung abgeleitete Nachbarschaftstabelle erstellt. Die Anordnung der APs (gekennzeichnet durch ihre im Bereich zwischen 11 und 75 liegenden Nummern) im Verlauf des Flures eines Bürogebäudes ist in Abbildung 6 auf der rechten Seite zu sehen. Links in der Abbildung ist die Matrix der Wahrscheinlichkeit gleichzeitiger Sichtbarkeit von jeweils zwei APs dargestellt: Während die Werte auf der Diagonalen jeweils trivialerweise $100 \%$ betragen, ist es höchst unwahrscheinlich, dass ein Terminal im Bereich des AP 60 (am Ende des südlichen Flurs) auch einen AP im nördlichen Bereich des Gebäudes ,sieht“: Wie aus der dritten Zeile (AP 60) in der Tabelle zu sehen, empfängt das Terminal bis auf AP 29, der dem südlichen Flur am nächsten liegt (2,6\%), nur Signale der APs im gleichen Flur (also AP 27 und AP 68). Des Weiteren erkennt man die Asymmetrie der Tabelle: Ein Terminal im Bereich von AP 60 wird zwar zu 


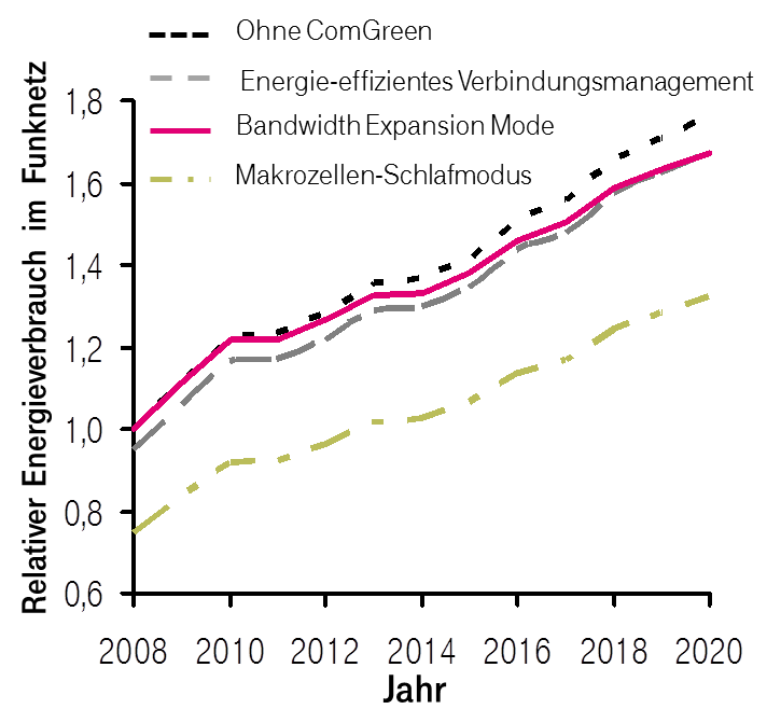

Abb. 9. Erwartete relative Entwicklung des Energieverbrauches im Mobilfunk innerhalb von 12 Jahren für verschiedene Maßnahmen zur Lastadaption.

$67.5 \%$ auch von AP 27 versorgt, umgekehrt ist für ein Terminal im Sichtbereich von AP 27 die Wahrscheinlichkeit mit $31,7 \%$ nicht einmal halb so groß, von AP 60 versorgt werden zu können, da AP 27 mit Lage in der westlichen Flurecke zu deutlich mehr Terminals nach Norden hin Verbindung aufnehmen kann.

Für energieeffizientes Verbindungsmanagement (energy efficient connectivity management) hat die Auswertung einer entsprechenden Simulation ergeben, dass Effizienzgewinne zwischen 5 und 9\% möglich sind. Die Energieeinsparung hängt dabei von der ausgewählten Strategie ab und variiert je nach Szenario: Simulationsergebnisse für eine Verkehrsverteilung, wie sie in einer typischen Innenstadt erwartet wird (z.B. mit dichter Bebauung und Fußgängerzonen, d.h. tagsüber hohe Anforderungen, nachts kaum Bedarf an breitbandiger Mobilfunkversorgung), werden in Abbildung 7 gezeigt. Die aus der links dargestellten Leistungsaufnahme im Tagesverlauf bestimmte Energieeinsparung bei der vorgeschlagenen Strategie (rot, „Proposed Policy $3_{\text {on+off }}$ ") gegenüber der ursprünglichen (magenta, ,Original Policy“) ergibt einen Effizienzgewinn von $5.5 \%$. In der Kurve auf der rechten Seite ist der durch mangelnde Kapazität bedingte Qualitätsverlust dargestellt. Für die wiederum durch die rote Kurve repräsentierte Strategie ist im Gegensatz zu anderen Strategien keine Verschlechterung zu erkennen. Für andere Szenarien ergeben sich leicht abweichende Werte - so ist der Effizienzgewinn für eine vorstädtische Gegend $5.8 \%$, in ländlicher Umgebung werden $8.6 \%$ erzielt, und bei einem Gebiet mit hohem Bedarf (sogenannter „hotspot“) lassen sich $6.8 \%$ Einsparung erreichen.

Die experimentelle Implementierung eines Optimierungsalgorithmus sowie die daraus erhaltenen Ergebnisse werden in (Gomez et al., 2013) detailliert beschrieben. Für ein Netz von 46 WLAN-Zugangsknoten zur Versorgung von 20 aktiven Endgeräten wurden umfangreiche Messungen durchgeführt. Die sowohl in- wie aushäusig positionierten WLANAPs wurden zur Messung der typischen Konfigurationsparameter und der für verschiedene Szenarien erzielbaren Energieeinsparung genutzt. Dabei benötigen die für einen Betrieb im Freien konzipierten und auf zwei Funkfrequenzen operierenden robusteren Outdoor-APs im Schnitt etwa doppelt so viel Energie im Vergleich zu den ,einfachen“, für IndoorBetrieb gedachten APs. Die Wirksamkeit des entwickelten Algorithmus „Morfeo“ zeigte sich durch Vergleich der Messungen mit und ohne eingeschaltetem Energie-Optimierer (EO), die in Abbildung 8 dargestellt sind.

Die mittlere Leistungsaufnahme wurde für Zugangspunkte (Wireless Access Device, WAD) im Haus (indoor) und im Freien (outdoor) für vier verschiedene Szenarien gemessen - jeweils im Normalbetrieb (,EO off“) und bei Optimierung (,EO on“). Die Szenarien unterscheiden sich nach der aufkommenden Verkehrslast: (a) ohne Datenverkehr, d.h. im sog. , idle mode“, (b) bei gleichmäßig konstantem Datenbedarf der Endgeräte, (c) dto., aber mit aktiver Lastverteilung, d.h. der Datenverkehr von und zu Zugangspunkten, die in einen Schlafmodus geschaltet werden, muss zu einem anderen WAD transferiert werden (Handover), (d) der Datenverkehr wird willkürlich erhöht, so dass WADs dynamisch ab- und angeschaltet werden. Durch die häufiger stattfindenden Rekonfigurationen ist die Energieeinsparung im letzteren Fall mit 9-25\% deutlich geringer als im statischen Fall, wo 20-35\% erzielt werden (siehe Abbildung 8b). Am größten ist die Einsparung erwartungsgemäß, wenn kein Datenverkehr fließt (Abbildung 8a). Es zeigt sich allerdings durchgängig, dass im Indoor-Betrieb von WLAN-Zugangsnetzen mehr Energie eingespart werden kann als beim Betrieb im Freien. Die Messungen belegen, dass in Abhängigkeit vom Szenario im Durchschnitt bei WLAN-Netzen durch den Morfeo-Algorithmus 9-36\% an Energie eingespart werden kann.

\section{Wertung und Ausblick}

Wie bereits einleitend genannt, muss (nicht nur in Deutschland) von einem in Zukunft weiter kontinuierlich steigenden Bedarf nach hochratiger mobiler Datenübertragung ausgegangen werden. Bei Annahme einer Steigerung von 50\% im Jahresmittel wurden die relative Steigerung im Energieverbrauch im Mobilfunksektor und die durch ComGreenMaßnahmen erwartete Einsparung abgeschätzt. Abbildung 9 zeigt die Auswirkung verschiedener Verfahren im Vergleich zum Normalfall (,ohne ComGreen“; punktierte Kurve), wobei bereits die technologische Weiterentwicklung der Netzkomponenten (hardware) einberechnet wurde. So wurde über alle Technologien gemittelt - eine jährliche Effizienzsteigerung von $3 \%$ und ein alle acht Jahre stattfindender 
Geräteaustausch angenommen. Durch energieeffizientes Verbindungsmanagement (gestrichelt) lassen sich durchgängig zwischen 5 und $9 \%$ Einsparung erzielen, da die Last in einzelnen Zellen immer unterschiedlich sein wird. Die mit dem Bandwidth Expansion Mode (BEM) erzielbare Einsparung beträgt bis zu $25 \%$, lässt sich allerdings nur bei einer der z.Z. genutzten Funktechniken (LTE) anwenden. Da der Anteil der Stationen dieser neuesten Technik stetig zunimmt, wird sich die Einsparung (rote durchgezogene Kurve) entsprechend erhöhen. Durch Makrozellen-Schlafmodus können 20-30\% vom Energieverbrauch eingespart werden. Dieses Verfahren wird in vollem Umfang allerdings wohl erst in wenigen Jahren einsetzbar sein, da entsprechende Technik zum raschen Einschalten von Stationen noch nicht verfügbar ist.

Die Darstellung in Abbildung 9 ist nicht als additiv zu betrachten - einzelne Verfahren sind weniger effizient wenn andere bereits genutzt werden: So ist der Gewinn beim Schlafmodus für LTE geringer, wenn bereits BEM zum Energiesparen genutzt wird. Analog lassen sich durch connectivity management geringere Einsparungen erzielen, wenn manche Zellen bereits im Schlafmodus sind und daher nicht zur Auswahl stehen. Insgesamt kann allerdings bei geschickter Anwendung von Lastadaptivität im Mobilfunk mit einer auf wenige \% begrenzten Erhöhung beim Energieverbrauch gerechnet werden - vor allem, wenn man einbezieht, dass Aktivitäten zur Steigerung der Energieeffizienz weltweit weitergehen und der Druck auf Netzbetreiber und Hersteller absehbar nicht sinken wird.

\section{Zusammenfassung}

Moderne Telekommunikationsnetze erlauben durch ihr breites Spektrum an verfügbaren Diensten und die zunehmende Verbreitung und Nutzbarkeit sowohl stationär (Festnetz) als auch unterwegs (Mobilfunk) eine universelle Versorgung von Privat- und Geschäftskunden mit ITK-Anwendungen jeglicher Art. Vielfach können Ressourcen wie Zeit, Rohstoffe und Energie eingespart werden - wodurch auch ein erheblicher Beitrag zum Klimaschutz geleistet werden kann. So wird allein in der Gebäudetechnik z.B. der durch Smartphone-gestützte Analyse identifizierte einsparbare Anteil am Gesamtenergieverbrauch weltweit auf $3.7 \%$ geschätzt (Leslie et al., 2012; Jing und Nayel, 2013). Dagegen wird der durch Telekommunikation verursachte Energiebedarf auf ca. 2-5\% angesetzt - in der Tendenz allerdings steigend. Die für den Betrieb der Netze wie auch der Endgeräte benötigte Energie sollte den positiven Effekt aber nicht konterkarieren.

Daher wurden im Rahmen des Förderprogramms der Bundesregierung IT2Green Maßnahmen zur Energieeinsparung bei IKT erforscht. Das Projekt ComGreen hat sich mit Methoden zur Reduzierung des Energieverbrauchs in Mobilfunknetzen befasst. Die Ergebnisse des im Mai 2014 abgeschlos- senen Projektes zeigen, dass über die aufgrund der technischen Weiterentwicklung in der Mobilfunk-Systemtechnik erzielbare Effizienzsteigerung hinaus weitere Einsparungen im Bereich zwischen 25 und $40 \%$ realistisch sind. Die Wirksamkeit der vorgeschlagenen Maßnahmen zum kontextbasierten lastadaptiven Betrieb von Mobilfunksystemen wurde in theoretischen Untersuchungen und durch Systemsimulationen quantifiziert sowie deren Machbarkeit experimentell nachgewiesen.

Danksagung. Die Autoren bedanken sich bei den Konsortialpartnern des ComGreen-Projektes für die sehr gute Zusammenarbeit und beim BMWi sowie dem DLR für die Forschungsförderung und Projekt-begleitende Unterstützung. Unseren Kollegen und Studenten sei insbesondere für die über das Projekt hinausgehende Unterstützung bei Implementierungen und Messungen gedankt. Vielen Dank auch an Christoph Lange und den unbekannten Reviewer für die hilfreichen Verbesserungsvorschläge.

Herausgeber: J. Anders

Reviewed by: two anonymous referees

\section{Literatur}

3GPP: TR 32.826 Study on ES Management (ESM) (Rel. 10), 2010. 3GPP: TR 36.927, Potential solutions for energy saving for EUTRAN (Rel. 10), 2011.

Bayer, N., Sivchenko, D., Einsiedler, H.-J., Roos, A., Uzun, A., Göndör, S., and Küpper, A.: Energy optimisation in heterogeneous multi-RAT networks, 15 th International Conference on Intelligence in Next Generation Networks (ICIN), Oktober 2011, 139-144, 2011.

Bayer, N., Gomez, K., Sengul, C., von Hugo, D., Göndör, S., and Uzun, A.: Load-Adaptive Networking for Energy-Efficient Wireless Access, eingereicht bei Computer Networks, Special Issue on Green Communications, März, 2014.

BMWi: IT2Green im Kontext von Green IT, verfügbar unter: http: //www.it2green.de/de/1252.php, zuletzt abgerufen: Juni 2014.

BNetzA: Jahresberichte der Bundesnetzagentur, verfügbar unter: http://www.BNetzA.de, zuletzt abgerufen: Juli 2014.

Cisco: Cisco Visual Networking Index: Global Mobile Data Traffic Forecast Update, 2013-2018, Feb. 2014, verfügbar unter: http: //www.cisco.com/c/en/us/solutions/collateral/service-provider/ visual-networking-index-vni/white_paper_c11-520862.pdf, zuletzt abgerufen: Juni 2014.

ComGreen: Deliverable Report D2.3, Final Evaluation of the Algorithms in Single RAT and Multi RAT, 8-14, Februar, 2013 (nur Englisch).

ComGreen: Projekt ComGreen - Kurzbeschreibung, verfügbar unter: http://www.it2green.de/de/771.php bzw. Internetpräsenz des Projektes auf http://www.communicate-green.de (nur Englisch), zuletzt abgerufen: Juni 2014.

Correia, L. M., Zeller, D., Blume, O., Ferling, D., Jading, Y., Goìdor, I., Auer, G., and Van der Perre, L.: Challenges and Enabling Technologies for Energy Aware Mobile Radio Networks, IEEE Comm. Soc. Mag., Nov. 2010, http://ieeexplore.ieee.org/xpl/ 
articleDetails.jsp?arnumber=5621969 (zuletzt abgerufen März 2015), 2010.

De Domenico, A., Calvanese Strinati, E., and Capone, A.: Enabling Green cellular networks: A survey and outlook, Computer Communications Volume 37, 5-24, 2014.

Deutsche Telekom AG: Nachhaltigkeit to go - Von der Straßendemo ins Smartphone, 2011, verfügbar unter: http://www.studie-life.de/wp-content/uploads/2011/11/

Report-110530-Nachhaltigkeit-to-go.pdf, 2011.

E3: https://ict-e3.eu/project/technical_highlights/4_ca/ca_2009_ 04.html (zuletzt abgerufen März 2015), 2009.

ETSI MCC: 3GPP Green activities / Energy Saving V0.0.9, 2012.

Gomez, K. M., Bayer, N., and Sengul, C.: Energy-Saving Framework for Wireless Access Infrastructures, Proceedings of the 2013 IEEE Online Conference on Green Communications (IEEE Online GreenComm 2013), IEEE, USA, Oktober, 2013.

GreenTouch: White Paper, Green Meter Research Study: Reducing the Net Energy Consumption in Communications Networks by up to $90 \%$ by 2020 , Juni 2013 , verfügbar unter: http://www. GreenTouch.org, 2013.

Hsieh, C.-H., Chao, S.-L., Chen, Y.-Y., Yang, C.-C., and Wie, H.-Y.: Smartphone Traffic Engineering for Energy Efficient Communications: Design and Experimental Evaluation, Wireless Personal Communications, 74, 1179-1196, 2014.

Jing, W. and Nayel, M.: Energy audits and energy-saving potential analysis of the science building at Xian Jiaotong-Liverpool university in Suzhou, Applied Mechanics and Materials, 291-294, 1044-1049, 2013.

Kaus, M.: Development, implementation and evaluation of new optimization concepts for energy saving in heterogeneous radio access network, Diplomarbeit, Technische Hochschule Mittelhessen, Gießen-Friedberg, April, 40-43, 2012.

Lange, C., Kosiankowski, D., Betker, A., Simon, H., Bayer, N., von Hugo, D., Lehmann, H., and Gladisch, A.: Energy Efficiency of Load-Adaptively Operated Telecommunication Networks, IEEE/OSA J. Lightw. Technol. 32, 571-590, 2014.
Le Rouzic, E., Indre, R.-M., Chiaraviglio, L., Musumeci, F., Pattavina, A., Lopez Vizcaino, J., Yabin Y., Van Heddeghem, W., Bianco, A., Bonetto, E., Meo, M., Jimenez, F., Idzikowski, F., and Cuevas, R.: TREND big picture on energy-efficient backbone networks, in: 24th Tyrrhenian International Workshop on Digital Communications - Green ICT (TIWDC), September, 2013.

Leslie P., Pearce J.M., Harrap R., and Daniel S.: The application of smartphone technology to economic and environmental analysis of building energy conservation strategies, International Journal of Sustainable Energy, 31, 295-311, 2012.

OECD: Measuring the Relationship between ICT and the Environment, Juli, 2009.

OPERA-NET: www.opera-net.org, web site des Projektes OPERANET, zuletzt abgerufen: Juni 2014.

Scheck, H.-O.: User equipment receiver sensitivity: The forgotten mobile network efficiency factor, ETSI Workshop on Environmental Impact Assessment and Energy Efficiency, Oktober 2013, Athen, Griechenland, online verfügbar unter: http://docbox.etsi. org/Workshop/, 2013.

The Climate Group/GeSI: Smart 2020 report, Enabling the low carbon economy in the information age, 2008, verfügbar unter: http://www.theclimategroup.org/assets/resources/ publications/Smart2020Report.pdf (zuletzt abgerufen: im Juli 2014), 2008.

Uzun, A., Küpper, A., and Einsiedler, H.-J.: COMMUNICATE GREEN - Energy Efficient Mobile Communication, in: Proceedings of the 1st International Conference on Pervasive and Embedded Computing and Communication Systems (PECCS 2011), 302-305, SciTePress, Vilamoura, Algarve, Portugal, März, 2011.

Uzun, A., von Hoffen, M., and Küpper, A.: Enabling Semantically Enriched Data Analytics by Leveraging Topology-based Mobile Network Context Ontologies, in: Proceedings of the 4th International Conference on Web Intelligence, Mining and Semantics (WIMS 2014), ACM, Thessaloniki, Greece, Juni, 2014. 